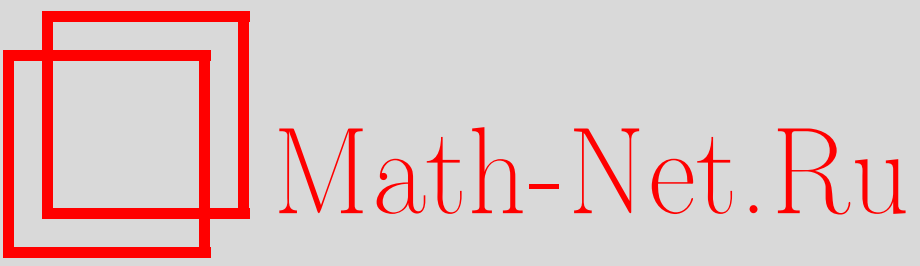

Н. И. Гусева, Пространства над алгебрами с евклидовой метрикой, Итоги науки и техн. Сер. Соврем. мат. и ее прил. Темат. обз., 2020, том 179, 10-15

DOI: https://doi.org/10.36535/0233-6723-2020-179-10-15

Использование Общероссийского математического портала Math-Net.Ru подразумевает, что вы прочитали и согласны с пользовательским соглашением

http://www.mathnet.ru/rus/agreement

Параметры загрузки:

IP : 54.205 .225 .156

26 апреля 2023 г., 17:19:14 
ИТОГИ НАУКИ И ТЕХНИКИ.

Современная математика и ее приложения.

Тематические обзоры.

Том 179 (2020). C. $10-15$

DOI: $10.36535 / 0233-6723-2020-179-10-15$

УДК 514.169

\title{
ПРОСТРАНСТВА НАД АЛГЕБРАМИ С ЕВКЛИДОВОЙ МЕТРИКОЙ
}

\author{
(C) 2020 г. $\quad$ Н. И. ГУСЕВА
}

\begin{abstract}
АннотАция. В статье дан новый подход к вещественной реализации пространств над алгебрами, при котором пространство над алгеброй и пространство его овеществления имеют одинаковую размерность, и приведены несколько примеров из евклидовой геометрии, которые иллюстрируют предложенную вещественную реализацию пространств над линейными алгебрами.
\end{abstract}

Ключевые слова: пространство над алгеброй, структура алгебры в линейном пространстве, комплексное линейное пространство, размерность, метрика, вещественная реализация пространств.

\section{SPACES OVER ALGEBRAS WITH EUCLIDEAN METRIC}

\section{(c) 2020 N. I. GUSEVA}

\begin{abstract}
In this paper, we propose a new approach to the real realization of spaces over algebras, in which a space over an algebra and the space of its realizations have the same dimension. We also give several examples from Euclidean geometry, which illustrate this real realization of spaces over linear algebras.
\end{abstract}

Keywords and phrases: space over algebra, structure of an algebra in a linear space, complex linear space, dimension, metric, real realization of a space.

AMS Subject Classification: 13K05, 51N20, 51M04

Пространства над алгебрами занимают как бы «промежуточное положение» между линейными (векторными) пространствами над тем или иным полем и модулями над различными кольцами.

Изучение такого объекта восходит к работам российского геометра А. П. Котельникова и немецкого математика Э. Штуди, и впоследствии развивалось разными геометрическими школами, как отечественными, так и зарубежными. В частности, большой вклад в развитие теории пространств над алгебрами внесли математики Московской и Казанской геометрических школ Б. А. Розенфельд, А. П. Широков, В. В. Вишневский, В. В. Шурыгин и др. (см. [3, 5]).

Линейные алгебры представляют собой частный случай колец, и с этой точки зрения пространства над алгебрами являются разновидностью модулей. Но особенностью пространств над алгебрами, отличающей этот объект от общей конструкции модуля, является возможность толкования пространств над алгебрами как линейных пространств над полем, над которым определена соответствующая линейная алгебра. При этом размерность линейного пространства, представляющего пространство над некоторой алгеброй, будет произведением размерности алгебры на размерность данного пространства над алгеброй. А структура алгебры в линейном пространстве, представляющем пространство над алгеброй, определяется набором линейных операторов, произведение которых повторяет произведение базисных элементов данной алгебры. И если поле, над которым задана линейная алгебра, будет полем вещественных чисел, то переход от пространства над алгеброй к вещественному векторному пространству называется овеществлением данного пространства над алгеброй. 
Простейший пример пространств над алгебрами доставляют комплексные линейные пространства, так как поле комплексных чисел является двухмерной линейной алгеброй. Таким образом, линейное комплексное пространство $\mathbb{C}^{n}$ можно представить вещественным пространством $\mathbb{R}^{2 n}$, в котором задан оператор $J: \mathbb{R}^{2 n} \rightarrow \mathbb{R}^{2 n}$, удовлетворяющий тождеству $J^{2}=\mathrm{id}$, где id - тождественный оператор.

В общем случае, если линейная алгебра $\boldsymbol{A}_{m}$ задана некоторым своим базисом $\varepsilon_{1}, \boldsymbol{\varepsilon}_{2}, \ldots, \boldsymbol{\varepsilon}_{m}$, элементы которого удовлетворяют структурным тождествам $\varepsilon_{k} \cdot \varepsilon_{h}=\gamma_{k h}^{r} \varepsilon_{r}$, то овеществлением пространства над данной алгеброй $\boldsymbol{E}_{n}\left(\boldsymbol{A}_{m}\right)$, где $\operatorname{dim}_{\boldsymbol{A}_{m}} \boldsymbol{E}_{n}\left(\boldsymbol{A}_{m}\right)=n$, будет линейное вещественное пространство $\mathbb{R}^{n m}$ с заданной на нем системой операторов $J_{k}$, удовлетворяющих условию $J_{k} \cdot J_{h}=\gamma_{k h}^{r} J_{r}$.

Такое толкование перехода от пространств над алгебрами к вещественным линейным пространствам можно назвать классическим, так как по сути оно восходит к геометрической интерпретации комплексных чисел, которую обыкновенно называют интерпретацией Гаусса. (Хотя, справедливости ради, надо сказать, что представление комплексных чисел точками плоскости было дано на рубеже XVIII-XIX вв. независимо друг от друга целым рядом математиков, и зачастую раньше работ Гаусса на эту тему).

Описанная выше классическая интерпретация пространств над алгебрами как вещественных векторных пространств послужила основой для различных геометрических приложений пространств над алгебрами, так как придавала абстрактной алгебраической конструкции определенный геометрический смысл. Однако классическое толкование пространств над алгебрами как вещественных векторных пространств имеет ряд существенных недостатков, среди которых можно выделить следующие. Во-первых, овеществление пространств над алгебрами кратно увеличивает размерность пространства, вследствие чего теряется наглядность. Например, овеществление комплексной плоскости дает четырехмерное вещественное пространство, которое с трудом поддается нашей интуиции. Во-вторых, при переходе от пространств над алгебрами к вещественным пространствам искажается смысл многих геометрических терминов. Например, прямой в пространстве над алгеброй размерности $m$ будет $m$-мерная плоскость в вещественном пространстве, представляющем данное пространство над алгеброй. В-третьих, введение дополнительной структуры в вещественном пространстве, представляющем пространство над алгеброй, требует согласование этой структуры с действием линейных операторов, соответствующих базисным элементам алгебры, что не всегда возможно и т. д.

Для того чтобы устранить эти недостатки классической интерпретации пространств над алгебрами, нужна новая интерпретация, которая представляла бы $n$-мерное пространство над линейной алгеброй любой размерности $m$ на $n$-мерном вещественном пространстве. Такая интерпретация пространств над алгебрами в начале восьмидесятых годов была дана М. П. Бурлаковым для представления различных физических полей в пространственно-временных многообразиях. Ее основная идея заключалась в том, чтобы вектор $n$-мерного пространства над $m$-мерной алгеброй представлять в $n$-мерном вещественном пространстве суммой $m$ вещественных векторов, каждый из которых помечен базисным элементом линейной алгебры как множителем. При этом векторы, помеченные разными базисными элементами алгебры, считаются линейно независимыми в $n$ мерном вещественном пространстве.

Имеется несколько возможных реализаций этой идеи (см. [4]). Например, набор $m$ вещественных векторов, формальная сумма которых представляет вектор в $n$-мерном пространстве над $m$-мерной алгеброй, можно считать расположенным в $n$-мерных плоскостях, помещенных параллельно в некотором аффинном пространстве размерности $N>n$. При этом принадлежность того или иного вектора из этого набора к определенной плоскости отмечается умножением на базисный элемент алгебры. А системы координат на всех плоскостях согласованы так, что векторы, отличающиеся лишь алгебраическим сомножителем из базиса алгебры, будут иметь одинаковые вещественные координаты. Такую интерпретацию пространств над алгебрами естественно называть многолистной. Эта интерпретация пространств над алгебрами использовалась М. П. Бурлаковым для описания «параллельных вселенных» в пространственно-временных многообразиях. 
Другая интерпретация трактует вектор $n$-мерного пространства над $m$-мерной алгеброй как формальную линейную комбинацию $m$ вещественных векторов в $n$-мерном вещественном пространстве, коэффициентами которой являются базисные элементы данной линейной алгебры. Наглядно векторы, умноженные на базисные элементы алгебры, можно представлять окрашенными в различные цвета, и потому такую интерпретацию пространств над линейными алгебрами естественно называть «цветной». Эта интерпретация имеет свой аналог в теоретической физике, где одинаковые кварки (кварки одного «аромата») наделяют одним из трех «цветов», чтобы удовлетворить статистике Ферми, запрещающей находиться в одном состоянии двум одинаковым частицам с полуцелым спином.

Впрочем, по сути обе интерпретации пространств над алгебрами совпадают, так как принадлежность вектора какому-либо листу многолистного пространства можно заменить его окраской в соответствующий цвет. И такая замена изоморфна относительно операций над формальными суммами вещественных векторов, имеющими сомножителями базисные элементы алгебры.

Предложенная многолистная (или многоцветная) интерпретация пространств над алгебрами свободна от указанных выше недостатков классической интерпретации. Все геометрические объекты располагаются теперь в вещественном пространстве той же размерности, что и алгебраическая размерность пространства над алгеброй. При этом смысл геометрических объектов остается одинаковым и в пространствах над алгебрами, и в их многолистной интерпретации. Например, линия в пространстве над $m$-мерной алгеброй будет линией и в вещественном пространстве многолистной интерпретации, правда, линия в многолистной интерпретации будет иметь $m$ ветвей. Наконец, в многолистной интерпретации пространств над алгебрами любая геометрическая структура, заданная в вещественном пространстве, автоматически переносится на случай пространств над алгебрами.

Иллюстрацией к сказанному могут быть пространства над алгебрами с евклидовой метрикой. Пусть $\boldsymbol{E}_{n}$ - вещественное евклидово пространство, $\boldsymbol{e}_{1}, \boldsymbol{e}_{2}, \ldots, \boldsymbol{e}_{n}$ - какой-либо ортонормированный базис этого пространства, а $\boldsymbol{A}_{m}$ - вещественная линейная алгебра с базисом $\boldsymbol{\varepsilon}_{1}, \boldsymbol{\varepsilon}_{2}, \ldots, \boldsymbol{\varepsilon}_{m}$. Тогда базисом пространства над алгеброй $\boldsymbol{E}_{n}\left(\boldsymbol{A}_{m}\right)$ будет набор элементов $\boldsymbol{\varepsilon}_{\alpha} \cdot \boldsymbol{e}_{k}$, так что любой алгебраический вектор $\boldsymbol{x} \in \boldsymbol{E}_{n}\left(\boldsymbol{A}_{m}\right)$ можно представить в виде $\boldsymbol{x}=x_{1} \varepsilon_{1}+x_{2} \varepsilon_{2}+\ldots+x_{m} \boldsymbol{\varepsilon}_{m}$, где $x_{1}, x_{2}, \ldots, x_{m} \in \boldsymbol{E}_{n}$. Таким образом, скалярное произведение алгебраических векторов $\boldsymbol{x}, \boldsymbol{y} \in$ $\boldsymbol{E}_{n}\left(\boldsymbol{A}_{m}\right)$ будет иметь вид

$$
(\boldsymbol{x}, \boldsymbol{y})=\sum\left(x_{\alpha}, y_{\beta}\right) \gamma_{\alpha \beta}^{\tau} \boldsymbol{\varepsilon}_{\tau},
$$

где $\left(x_{\alpha}, y_{\beta}\right)$ - скалярные произведения векторов $x_{\alpha}, y_{\beta} \in \boldsymbol{E}_{n}$, а $\gamma_{\alpha \beta}^{\tau}$-структурные константы алгебры $\boldsymbol{A}_{m}$, т.е. $\boldsymbol{\varepsilon}_{\alpha} \cdot \boldsymbol{\varepsilon}_{\beta}=\gamma_{\alpha \beta}^{\tau} \varepsilon_{\tau}$.

Из (1) видно, что в многолистной интерпретации пространства над алгеброй $\boldsymbol{E}_{n}\left(\boldsymbol{A}_{m}\right)$ вычисление скалярного произведения сводится к вычислению скалярных произведений вещественных векторов в метрике пространства $\boldsymbol{E}_{n}$ и произведений базисных элементов линейной алгебры $\boldsymbol{A}_{m}$.

Предложенная многолистная (или цветная) интерпретация пространств над алгебрами необычна, поэтому естественно протестировать ее на простых фактах, допускающих проверку элементарными средствами. Примером этому может служить так называемая теорема о вставленном треугольнике (см. [2]).

Пусть $A B C$-треугольник на евклидовой плоскости и пусть точки $A_{1}, B_{1}, C_{1}$ делят стороны треугольника $A B C$ в отношении $\nu / \mu$, т.е.

$$
\frac{A A_{1}}{A_{1} C}=\frac{B B_{1}}{B_{1} A}=\frac{C C_{1}}{C_{1} B}=\frac{\nu}{\mu}
$$

где для определенности $\mu+\nu=1$. Тогда

$$
\frac{\left|A_{1} B_{1}\right|^{2}+\left|B_{1} C_{1}\right|^{2}+\left|C_{1} A_{1}\right|^{2}}{|A B|^{2}+|B C|^{2}+|C A|^{2}}=\mu^{3}+\nu^{3},
$$

т.е. отношение суммы квадратов длин сторон вставленного треугольника $A_{1} B_{1} C_{1}$ к сумме квадратов длин сторон исходного треугольника $A B C$ равно $\mu^{3}+\nu^{3}$. 
Для доказательства рассмотрим комплексную плоскость $\mathbb{C}^{2}$ и на ее вещественном листе возьмем радиус-векторы $a, b, c$ точек $A, B, C$, которые соберем в один комплексный вектор $\boldsymbol{x}=a+\alpha_{3} b+\alpha_{3}^{2} c$, где

$$
\alpha_{3}=\cos \frac{2 \pi}{3}+i \sin \frac{2 \pi}{3} .
$$

Тогда скалярное произведение комплексного вектора $\boldsymbol{x}$ на сопряженный вектор $\overline{\boldsymbol{x}}=a+\alpha_{3}^{2} b+\alpha_{3} c$ даст половину сумму квадратов длин сторон треугольника $A B C$, так как

$$
\begin{gathered}
2(\boldsymbol{x}, \overline{\boldsymbol{x}})=2\left((a, a)+(b, b)+(c, c)+((a, b)+(b, c)+(c, a))\left(\alpha_{3}+\alpha_{3}^{2}\right)\right)= \\
=2((a, a)+(b, b)+(c, c)-(a, b)-(b, c)-(c, a))= \\
=((a-b),(a-b))+((b-c),(b-c))+((c-a),(c-a))=|A B|^{2}+|B C|^{2}+|C A|^{2} .
\end{gathered}
$$

Если теперь вектор $\boldsymbol{x}$ умножить на комплексное число $\beta=\mu+\nu \alpha_{3}$, то вещественными компонентами комплексного вектора

$$
\boldsymbol{y}=\beta \boldsymbol{x}=\left(\mu+\nu \alpha_{3}\right)\left(a+\alpha_{3} b+\alpha_{3}^{2} c\right)=(\mu a+\nu c)+\alpha_{3}(\mu b+\nu a)+\alpha_{3}^{2}(\mu b+\nu a)=a_{1}+b_{1} \alpha_{3}+c_{1} \alpha_{3}^{2}
$$

будут радиус-векторы $a_{1}, b_{1}, c_{1}$ точек $A_{1}, B_{1}, C_{1}$. Поэтому

$$
2(\boldsymbol{y}, \overline{\boldsymbol{y}})=\left|A_{1} B_{1}\right|^{2}+\left|B_{1} C_{1}\right|^{2}+\left|C_{1} A_{1}\right|^{2}
$$

Но $(\boldsymbol{y}, \overline{\boldsymbol{y}})=\beta \bar{\beta}(\boldsymbol{x}, \overline{\boldsymbol{x}})=\left(\mu^{3}+\nu^{3}\right)(\boldsymbol{x}, \overline{\boldsymbol{x}})$, откуда и следует тождество (2).

Тождество (2) можно обобщить на разные многоугольники (см. [2,4]). Например, если на сторонах любого пятиугольника $A B C D E$ взять такие точки $A_{1} B_{1} C_{1} D_{1} E_{1}$, что

$$
\frac{A A_{1}}{A_{1} E}=\frac{B B_{1}}{B_{1} A}=\frac{C C_{1}}{C_{1} B}=\frac{D D_{1}}{D_{1} C}=\frac{E E_{1}}{E_{1} D}=\frac{\nu}{\mu},
$$

где $\mu+\nu=1$, и ввести обозначения

$$
\begin{aligned}
& S_{0}=|A B|^{2}+|B C|^{2}+|C D|^{2}+|D E|^{2}+|E A|^{2}, \\
& S_{1}=|A C|^{2}+|B D|^{2}+|C E|^{2}+|D A|^{2}+|E B|^{2}, \\
& S_{0}^{\prime}=\left|A_{1} B_{1}\right|^{2}+\left|B_{1} C_{1}\right|^{2}+\left|C_{1} D_{1}\right|^{2}+\left|D_{1} E_{1}\right|^{2}+\left|E_{1} A_{1}\right|^{2}, \\
& S_{1}^{\prime}=\left|A_{1} C_{1}\right|^{2}+\left|B_{1} D_{1}\right|^{2}+\left|C_{1} E_{1}\right|^{2}+\left|D_{1} A_{1}\right|^{2}+\left|E_{1} B_{1}\right|^{2},
\end{aligned}
$$

то будет справедливо тождество

$$
\frac{\left(S_{0}^{\prime}-\phi^{2} S_{1}^{\prime}\right)\left(S_{1}^{\prime}-\phi^{2} S_{0}^{\prime}\right)}{\left(S_{0}-\phi^{2} S_{1}\right)\left(S_{1}-\phi^{2} S_{0}\right)}=\mu^{5}+\nu^{5}
$$

где $\phi=(1+\sqrt{5}) / 2-$ число золотого сечения (число Фидия).

Для доказательства тождества (3) надо взять комплексные векторы $\boldsymbol{x}=a+b \alpha_{5}+c \alpha_{5}^{2}+d \alpha_{5}^{3}+e \alpha_{5}^{4}$ и $\boldsymbol{y}=a+b \alpha_{5}^{2}+c \alpha_{5}^{4}+d \alpha_{5}+e \alpha_{5}^{3}$, где $a, b, c, d, e-$ вещественные радиус-векторы точек $A, B, C, D$, $E$, a

$$
\alpha_{5}=\cos \frac{2 \pi}{5}+i \sin \frac{2 \pi}{5}=\frac{\sqrt{5}-1}{4}+\frac{\sqrt{5+\sqrt{5}}}{2 \sqrt{2}} i .
$$

Нетрудно видеть, что

$$
\begin{gathered}
(\boldsymbol{x}, \overline{\boldsymbol{x}})=-\left(S_{0} \cos \frac{2 \pi}{5}+S_{1} \cos \frac{4 \pi}{5}\right), \quad(\boldsymbol{y}, \overline{\boldsymbol{y}})=-\left(S_{0} \cos \frac{4 \pi}{5}+S_{1} \cos \frac{2 \pi}{5}\right), \\
4(\boldsymbol{x}, \overline{\boldsymbol{x}})(\boldsymbol{y}, \overline{\boldsymbol{y}})=\phi^{-1}\left(S_{0}-\phi^{2} S_{1}\right)\left(S_{1}-\phi^{2} S_{0}\right) .
\end{gathered}
$$

Если теперь комплексные векторы $\boldsymbol{x}$ и $\boldsymbol{y}$ умножить соответственно на комплексные числа $\xi=$ $\mu+\nu \alpha_{5}$ и $\eta=\mu+\nu \alpha_{5}^{2}$, то получим комплексные векторы

$$
\begin{aligned}
& \boldsymbol{v}=a_{1}+b_{1} \alpha_{5}+c_{1} \alpha_{5}^{2}+d_{1} \alpha_{5}^{3}+e_{1} \alpha_{5}^{4}, \\
& \boldsymbol{u}=a_{1}+b_{1} \alpha_{5}^{2}+c_{1} \alpha_{5}^{4}+d_{1} \alpha_{5}+e_{1} \alpha_{5}^{3},
\end{aligned}
$$


где $a_{1}, b_{1}, c_{1}, d_{1}, e_{1}$ - вещественные радиус-векторы точек $A_{1}, B_{1}, C_{1}, D_{1}, E_{1}$, так что

$$
4(\boldsymbol{v}, \overline{\boldsymbol{v}})(\boldsymbol{u}, \overline{\boldsymbol{u}})=\phi^{-1}\left(S_{0}^{\prime}-\phi^{2} S_{1}^{\prime}\right)\left(S_{1}^{\prime}-\phi^{2} S_{0}^{\prime}\right)=\xi \bar{\xi} \eta \bar{\eta} 4(\boldsymbol{x}, \overline{\boldsymbol{x}})(\boldsymbol{y}, \overline{\boldsymbol{y}}),
$$

откуда и следует тождество (3), так как $\xi \bar{\xi} \eta \bar{\eta}=\mu^{5}+\nu^{5}$.

Конечно, тождества (2) и (3) можно доказать и без привлечения комплексных векторов, но само происхождение этих тождеств связанно с интерпретацией комплексной плоскости с евклидовой метрикой как двулистной вещественной евклидовой плоскости. Другие примеры утверждений евклидовой планиметрии, которые просто доказываются с привлечением двулистной интерпретации комплексной плоскости с евклидовой метрикой, даны в [4].

Разумеется, не только евклидова метрика сохраняется при «пополнении» вещественного векторного пространства элементами той или иной линейной алгебры. Например, если аффинное вещественное пространство «пополнить» элементами какой-либо линейной алгебры, то аффинные свойства сохранятся для алгебраических векторов и других объектов аффинного пространства над данной алгеброй.

В качестве иллюстрации здесь можно привести известную теорему элементарной геометрии, которая носит имя Пьера Вариньона, и утверждает, что середины сторон произвольного четырехугольника являются вершинами параллелограмма. Хотя стандартное доказательство этого факта тривиально, целесообразно дать здесь его «алгебраическое» доказательство.

Пусть $A B C D$ - произвольный четырехугольник, $a, b, c, d$-радиус-векторы его вершин, тогда критерием того, что $A B C D$ есть параллелограмм, будет тождество $a-b=d-c$ или $a-b+c-d=0$. Рассмотрим теперь алгебраический вектор $\boldsymbol{x}=a+b \boldsymbol{\varepsilon}+c \varepsilon^{2}+d \varepsilon^{3}$, где $\varepsilon$ образующий элемент циклической алгебры четвертого порядка $\mathbb{R}_{4}(\varepsilon)$, базис которой образован единицей и элементами $\varepsilon, \varepsilon^{2}, \varepsilon^{3}$, а $\varepsilon^{4}=1$. Тогда критерий того, что $A B C D$ есть параллелограмм, можно переписать в следующем виде: $\eta \cdot \boldsymbol{x}=0$, где $\eta=1-\boldsymbol{\varepsilon}+\boldsymbol{\varepsilon}^{2}-\boldsymbol{\varepsilon}^{3}$, так как

$$
\eta \cdot \boldsymbol{x}=(a-b+c-d)\left(1-\varepsilon+\varepsilon^{2}-\varepsilon^{3}\right) .
$$

Умножив теперь алгебраический вектор $\boldsymbol{x}=a+b \boldsymbol{\varepsilon}+c \varepsilon^{2}+d \boldsymbol{\varepsilon}^{3}$, составленный для произвольного четырехугольника, на элемент $\rho=(1+\varepsilon) / 2$, получим алгебраический вектор

$$
\boldsymbol{y}=\rho \cdot \boldsymbol{x}=\frac{a+d}{2}+\frac{b+a}{2} \varepsilon+\frac{c+b}{2} \varepsilon^{2}+\frac{d+c}{2} \varepsilon^{3}=a_{1}+b_{1} \varepsilon+c_{1} \varepsilon^{2}+d_{1} \varepsilon^{3},
$$

где $a_{1}, b_{1}, c_{1}, d_{1}$-радиус-векторы середин сторон $A D, B A, C B, D C$. Так как $\eta \cdot \rho=0$, то $\eta \cdot \rho \cdot \boldsymbol{x}=\eta \cdot \boldsymbol{y}=0$, т.е. $A_{1} B_{1} C_{1} D_{1}$ - параллелограмм.

Алгебраическое доказательство теоремы Вариньона выглядит гораздо сложнее элементарного доказательства, использующего свойство средней линии. Однако приведенное выше доказательство подсказывает следующее обобщение теоремы Вариньона.

Пусть $A_{1} A_{2} A_{3} \ldots A_{2 n}$ - произвольный $2 n$-угольник; тогда центры масс последовательно взятых $n$-угольников, т.е. $n$-угольников $A_{1} A_{2} \ldots A_{n}, A_{2} A_{3} \ldots A_{n+1}$ и т. д., образуют $2 n$-параллелограмм, т.е. $2 n$-угольник, у которого противоположные стороны равны и параллельны.

Рассмотрим, например, шестиугольники. Критерием того, что шестиугольник $A_{1} A_{2} A_{3} A_{4} A_{5} A_{6}$ будет 6-параллелограммом, является выполнение тождеств

$$
a_{1}-a_{2}+a_{4}-a_{5}=0, \quad a_{2}-a_{3}+a_{5}-a_{6}=0, \quad a_{3}-a_{4}+a_{6}-a_{1}=0,
$$

где $a_{1}, a_{2}, a_{3}, a_{4}, a_{5}, a_{6}$ - радиус-векторы вершин данного шестиугольника (см. [1]). Этот критерий можно переписать так:

$$
\eta \cdot \boldsymbol{x}=\left(1-\varepsilon^{2}+\varepsilon^{3}-\varepsilon^{5}\right) \cdot\left(a_{1}+a_{2} \varepsilon+a_{3} \varepsilon^{2}+a_{4} \varepsilon^{3}+a_{5} \varepsilon^{4}+a_{6} \varepsilon^{5}\right)=0
$$

где $\varepsilon$-образующий элемент циклической алгебры шестого порядка $\mathbb{R}_{6}(\varepsilon)$. Действительно, нетрудно видеть, что

$$
\begin{aligned}
\eta \cdot \boldsymbol{x}=\left(a_{1}-a_{2}+a_{4}-a_{5}\right) & +\left(a_{2}-a_{3}+a_{5}-a_{6}\right) \varepsilon+\left(a_{3}-a_{4}+a_{6}-a_{1}\right) \varepsilon^{2}+ \\
& +\left(a_{1}-a_{2}+a_{4}-a_{5}\right) \varepsilon^{3}+\left(a_{2}-a_{3}+a_{5}-a_{6}\right) \varepsilon^{4}+\left(a_{3}-a_{4}+a_{6}-a_{1}\right) \varepsilon^{5} .
\end{aligned}
$$


Тогда, умножая вектор $\boldsymbol{x}=a_{1}+a_{2} \varepsilon+a_{3} \varepsilon^{2}+a_{4} \varepsilon^{3}+a_{5} \varepsilon^{4}+a_{6} \varepsilon^{5}$, составленный для любого шестиугольника, на $\rho=\left(1+\varepsilon+\varepsilon^{2}\right) / 3 \in \mathbb{R}_{6}(\varepsilon)$, получим вектор

$$
\boldsymbol{y}=b_{1}+b_{2} \varepsilon+b_{3} \varepsilon^{2}+b_{4} \varepsilon^{3}+b_{5} \varepsilon^{4}+b_{6} \varepsilon^{5},
$$

где $b_{1}, b_{2}, b_{3}, b_{4}, b_{5}, b_{6}$ - радиус-векторы барицентров треугольников $A_{1} A_{5} A_{6}, A_{2} A_{1} A_{6}, A_{3} A_{2} A_{1}$, $A_{4} A_{3} A_{2}, A_{5} A_{4} A_{3}, A_{6} A_{5} A_{4}$. Поскольку

$$
\eta \cdot \rho=\left(1-\varepsilon^{2}+\varepsilon^{3}-\varepsilon^{5}\right) \cdot\left(1+\varepsilon+\varepsilon^{2}\right) / 3=0,
$$

то шестиугольник $B_{1} B_{2} B_{3} B_{4} B_{5} B_{6}$ является 6 -параллелограммом.

Примеры, заимствованные из евклидовой геометрии и приведенные здесь, призваны показать полезность предложенной М. П. Бурлаковым многолистной (или цветной) интерпретации пространств над алгебрами. Простые примеры наиболее убедительны. Так, интерпретация комплексных чисел точками на евклидовой плоскости, предложенная на рубеже XVIII-XIX вв., была сразу принята большинством математиков, так как в рамках этой интерпретации можно было доказывать самые разнообразные теоремы и решать задачи евклидовой планиметрии. Хотя, разумеется, приложения многолистной интерпретации пространств над алгебрами не ограничены лишь евклидовой геометрией.

\section{СПИСОК ЛИТЕРАТУРЫ}

1. Бахман Ф., Шмидт Э. n-Угольники. - М.: Мир, 1973.

2. Бурлаков М. П. Гамильтоновы алгебры. - М.: Граф-Пресс, 2006.

3. Вишневский В. В., Широков А. П., Шурыгин В. В. Пространства над алгебрами. - Казань: КГУ, 1985.

4. Гусева Н. И., Бурлаков И. М., Бурлаков М. П. Элементарная геометрия в пространствах над алгебрами. - М.: Интеллект-Центр, 2017.

5. Розенфельд Б. А. Неевклидовы геометрии. - М.: ГИТТЛ, 1955.

Гусева Надежда Ивановна

Московский педагогический государственный университет;

Всероссийский институт научной и технической информации РАН, Москва

E-mail: ngus12@mail.ru 